\title{
A subsystem identification method based on the path concept with coupling strength estimation
}

\author{
F.X. Magrans ${ }^{* 1,2}$, J. Poblet-Puig ${ }^{\dagger 2}$ and A. Rodríguez-Ferran ${ }^{\ddagger 2}$ \\ ${ }^{1}$ Ingeniería para el Control del Ruido \\ ${ }^{2}$ Laboratori de Càlcul Numèric, E.T.S. d'Enginyers de Camins, Canals \\ i Ports de Barcelona, Universitat Politècnica de Catalunya
}

August 16, 2017

\begin{abstract}
For complex geometries, the definition of the subsystems is not a straightforward task. We present here a subsystem identification method based on the direct transfer matrix, which represents the first-order paths. The key ingredient is a cluster analysis of the rows of the powers of the transfer matrix. These powers represent high-order paths in the system and are more affected than low-order paths by damping.

Once subsystems are identified, the proposed approach also provides a quantification of the degree of coupling between subsystems. This information is relevant to decide whether a subsystem may be analysed in a computer model or measured in the laboratory independently of the rest or subsystems or not. The two features (subsystem identification and quantification of the degree of coupling) are illustrated by means of numerical examples: plates coupled by means of springs and rooms connected by means of a cavity.
\end{abstract}

Keywords: path, subsystem, transfer matrix, coupling

\section{Introduction}

The subdivision of a mechanical system into subsystems according to their vibroacoustic response is a task required in several modelling methods or in order to better understand the behaviour of the mechanical system. A clear example is Statistical

\footnotetext{
*correspondence: Berruguete 52, Vila Olímpica (Vall d'Hebron), E-08035 Barcelona, Spain, email: fxmagrans@icrsl.com

${ }^{\dagger}$ correspondence: UPC, Campus Nord B1, Jordi Girona 1, E-08034 Barcelona, Spain, e-mail: jordi.poblet@upc.edu

${ }^{\ddagger}$ correspondence: UPC, Campus Nord C2, Jordi Girona 1, E-08034 Barcelona, Spain, e-mail: antonio.rodriguez-ferran@upc.edu
} 
Energy Analysis (SEA, [1, 2]) that requires, as a preliminary step, the definition of subsystems which satisfy several physical properties (high modal density, equipartition of energy between modes, equal probability of mode excitation, weak coupling between subsystems [3]). Also from an experimental viewpoint it can be interesting to know which parts of a large system (train coach, building, airplane) can be tested in the laboratory isolated from the other parts and the results will still be meaningful. A proper subsystem identification determines the quality in the estimation of modal parameters in the experimental or operational modal analysis [4], a review of techniques for parameter and system identification from measured data can be found in [5]. Finally, the knowledge about subsystem plays also an important role in the transfer path analysis and can determine which is the more appropriate technique to apply as well as the quality of the solution $[6,7,8]$.

The splitting of the domain is sometimes performed by hand, mainly based on experience and intuition. However, more systematic methods have also been proposed. Some of them simply choose the subsystems according to the material: a glass pane, an aluminium panel, etc. [9, 10], other are based on energy models [11, 12, 13], regard the frequency response function of a system [14], or study the shape of some eigenfunctions of the system [15].

In these last two methods [14, 15], cluster algorithms are used. Cluster analysis is a general purpose and very powerful tool that groups sets of objects in a big population according to their similitude [16]. The data to be provided is: the group of objects, the parameters that characterise them, the comparison criteria and how to measure their similitude (distance).

In this work we apply cluster analysis [17] in order to automatically define the subsystems in a vibroacoustic problem. The core of the method is based on the transfer matrices and their powers. This is representative of transmission paths inside the system.

Another goal of the proposal is to quantify the coupling strength between subsystems. To do this we define the coupling strength as the error committed if coupling is disregarded and each subsystem is solved isolatedly from the rest of subsystems: the larger the error, the larger the coupling strength. We do not attempt to give a strict definition of "weak coupling", because whether coupling between subsystems may be disregarded or not depends on the type of analysis: a certain relative error may be admissible for an industrial pre-project but not for the final design.

Some brief background concepts and the core idea of the method are explained in Section 2. The theoretical aspects and approach are illustrated by the numerical examples of Section 3. The concluding remarks of Section 4 close the paper.

\section{Methodology}

Our approach to automatically identify the subsystems is based on a combination of the powers of the transfer matrix $\mathbf{T}$ and a standard cluster analysis. $\mathbf{T}^{k}$ is a representation of the transmission paths inside the mechanical system. Detailed developments can be found in $[18,19,20,21]$ and the main concepts required in this section are overviewed in Section 2.1. The main idea of the identification method is exposed in 
Section 2.3.1. This is done in a conceptual way that is sustained latter by the numerical examples of Section 3. Section 2.3.2 explains the relation between this path-based method and other more widely used criteria. Finally, an important aspect is addressed in Section 2.4: the estimation of the coupling strength between subsystems.

\subsection{Overview of path analysis}

The problem statement as well as a brief summary of the transfer matrix concept is exposed here. The main goal is to see that a solution of a linear system can be expressed by means of the superposition of multiple paths, that is, powers of the transfer matrix [18].

Assume that the mechanical system is properly described by the linear system of equations

$$
\mathrm{Ax}=\mathrm{b}
$$

where the system matrix $\mathbf{A}$ can be split into diagonal $\mathbf{D}$, strictly lower triangular $\mathbf{L}$ and upper triangular $\mathbf{U}$ matrices

$$
\mathbf{A}=\mathbf{D}+\mathbf{L}+\mathbf{U}
$$

Following the usual convention, all vectors are by default column vectors (for instance, $\mathbf{x}$ and $\mathbf{b})$.

The linear system in (1) can be then rewritten as

$$
\mathbf{x}=\mathbf{D}^{-1} \mathbf{b}+\mathbf{T x}
$$

where

$$
\mathbf{T}=-\mathbf{D}^{-1}(\mathbf{L}+\mathbf{U})
$$

is the transfer matrix.

From Eq. (3), the solution of system (1) may be expressed as

$$
\mathbf{x}=(\mathbf{I}-\mathbf{T})^{-1} \mathbf{D}^{-1} \mathbf{b}
$$

It has been proved in [18] that the inverse of the matrix $(\mathbf{I}-\mathbf{T})$ can be expressed as the limit of a matrix series as

$$
(\mathbf{I}-\mathbf{T})^{-1}=\lim _{m \rightarrow \infty}\left(\sum_{k=0}^{m-n} \mathbf{T}^{k}+\sum_{k=m-n+1}^{m} \gamma_{m-k} \mathbf{T}^{k}\right)
$$

The series in Eq. (6) is unconditionally convergent, without any constraint on the value of $\|\mathbf{T}\|$, if coefficients $\gamma_{m-k}$ are properly chosen. For $\|\mathbf{T}\| \geq 1$, Eq. (6) with the optimal choice of $\gamma_{m-k}$ is convergent, whereas the standard Neumann series with $\gamma_{m-k}=1$ diverges. For $\|\mathbf{T}\|<1$, on the other hand, both series are convergent, but the convergence is faster with the optimal $\gamma_{m-k}$. These aspects are discussed in detail in [18].

Eq. (6) as well as expressions for the coefficients $\gamma_{m-k}$ are the main results in [18]. They allow the generalisation of the path superposition idea to any linear mechanical 
system (and not only to those with convergent Neumann series). So, a theoretical procedure based on the path concept has now a solid basis. In the present research, this idea is applied to the formulation of an algorithm for automatic sub-structuring of mechanical systems.

\subsection{Subsystems and transfer matrices}

We define a subsystem as a set of nodes with similar path behaviour. The concept of node is very general, see [18]: it may refer to a point in a continuous system, to a degree of freedom associated to that point, to a mass in a discrete system of masses and springs, to a subdomain of a continuous system,... In a computational model, for instance, "node" refers to the actual nodes of the finite element mesh, whereas in the laboratory a "node" is a point of excitation or measurement.

The transfer matrix $\mathbf{T}$ describes the direct signal transmission between nodes (that is, the first-order paths). How this matrix is obtained depends on the context: in a computational model, for instance, $\mathbf{T}$ is computed from the system matrix $\mathbf{A}$, see Eq. (4), or from a few selected nodes, see Section 3.4, whereas in the laboratory $\mathbf{T}$ is determined experimentally.

The powers $\mathbf{T}^{k}$ of the transfer matrix represent the $k$-order paths. If a $k$-order path remains inside a strongly connected zone, it is not attenuated: the entries in $\mathbf{T}^{k}$ that relate nodes in the same subsystem are large. On the contrary, if a $k$-order path passes through weakly connected subsystems it is attenuated, and the associated entries in $\mathbf{T}^{k}$ are small.

\subsection{Subsystem identification by means of cluster analysis}

\subsubsection{How it works?}

Based on the above discussion about the information carried by $\mathbf{T}^{k}$, our approach to identify subsystems consists of three simple steps: 
1. Obtain (i.e. compute or measure) the transfer matrix $\mathbf{T}$. Iterative loop

Set $k=2$

2. Compute power $\mathbf{T}^{k}$ of the transfer matrix, $\mathbf{T}^{k}=\mathbf{T}^{k-1} \mathbf{T}$.

3. Perform a cluster analysis of the rows of $\mathbf{T}^{k}$.

Set $k=k+1$ and go to step 2 .

The iterative loop is stopped when the clustering process is stable, that is, when the clusters of rows do not change as the power $k$ increases. To attain a stable clustering in fewer iterations, power $k$ may jump $s$ units at each iteration, $\mathbf{T}^{k+s}=\mathbf{T}^{k} \mathbf{T}^{s}$. In the numerical example of Section 3.1, for instance, we have taken $s=5$.

The clustering algorithm is similar to the one described in [15], but with one important difference: the elements are simply the rows of $\mathbf{T}^{k}$, and there is no need to divide the problem domain into cells nor to compute energies associated to those cells. The clustering process is hierarchical; this means that the rows of $\mathbf{T}^{k}$ are grouped progressively into sets of rows according to their correlation distance. As this distance increases, small sets of rows are grouped into larger sets. This clustering process can be graphically depicted as a tree diagram or dendrogram, see Figs. 7 and 19. The output of the algorithm is i) the optimal number of clusters, corresponding to the widest range of correlation distance (i.e. the longest branches in the dendrogram) and $i$ ) the rows of $\mathbf{T}^{k}$ in each cluster. The clustering algorithm is described in the three companion videos of Fig. 1.

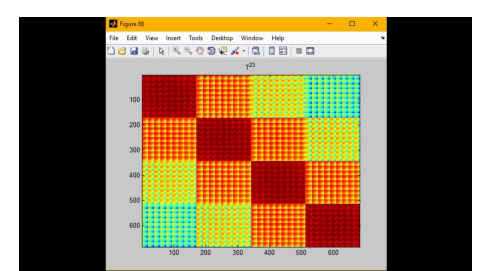

(a)

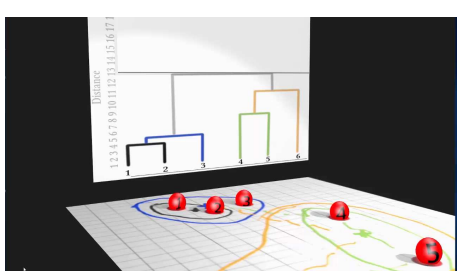

(b)

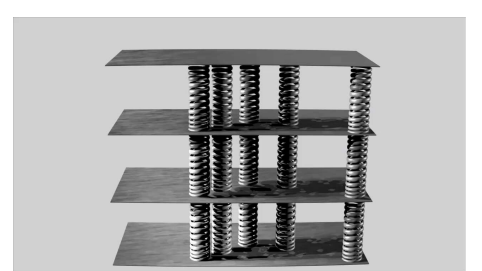

(c)

Figure 1: Screenshots of the three companion videos: (a) Relationship between the powers of the transfer matrix and the high-order paths; (b) Explanation of the dendrogram concept and the clustering process; (c) Illustration of the clustering algorithm by means of an example.

\subsubsection{Why it works?}

A simple explanation. The analysis is restricted here to systems composed of two subsystems. Consider first the case where these two subsystems are completely uncoupled, Fig. 2(a). The transfer matrix is

$$
\mathbf{T}=\left(\begin{array}{cc}
\mathbf{T}_{1} & \mathbf{0} \\
\mathbf{0} & \mathbf{T}_{2}
\end{array}\right)
$$

Note that the off-diagonal blocks in the transfer matrix (7) are zero because there is no coupling between subsystems 1 and 2. The transfer matrices $\mathbf{T}_{1}$ and $\mathbf{T}_{2}$ are 


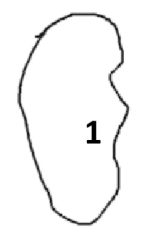

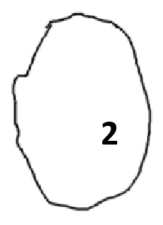

(a)

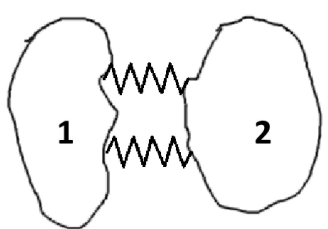

(b)

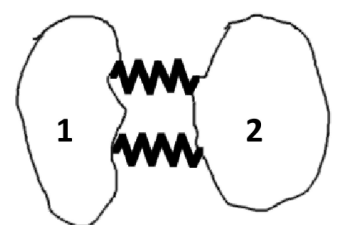

(c)

Figure 2: (a) Uncoupled subsystems; (b) Weakly coupled subsystems; (c) Strongly coupled subsystems

typically sparse because not all nodes in subsystem 1 (or 2) are directly connected, by means of a first-order path, to all nodes in subsystem 1 (or 2). The $k$ power of matrix $\mathbf{T}$ is

$$
\mathbf{T}^{k}=\left(\begin{array}{cc}
\mathbf{T}_{1}^{k} & \mathbf{0} \\
\mathbf{0} & \mathbf{T}_{2}^{k}
\end{array}\right)
$$

For $k$ large enough, the block $\mathbf{T}_{1}^{k}$ and $\mathbf{T}_{2}^{k}$ ( $k$-order paths in subsystems 1 and 2 ) are full matrices, so the sparsity pattern of matrix $\mathbf{T}^{k}$ is:

$$
\operatorname{pattern}\left(\mathrm{T}^{k}\right)=\left(\begin{array}{ccccccc}
\bullet & \bullet & \bullet & \bullet & & & \\
\bullet & \bullet & \bullet & \bullet & & & \\
\bullet & \bullet & \bullet & \bullet & & & \\
\bullet & \bullet & \bullet & \bullet & \bullet & \bullet & \bullet \\
& & & & \bullet & \bullet & \bullet \\
& & & & \bullet & \bullet & \bullet
\end{array}\right)
$$

A cluster analysis of the rows of $\mathbf{T}^{k}$ readily detects that there are two different types of rows, namely

$$
\text { ) and ( }
$$

and identifies the two subsystems. Note that this approach also works if the system nodes are not ordered (first nodes in subsystem 1, then nodes in subsystem 2). Indeed for an arbitrary ordering the sparsity pattern of $\mathbf{T}^{k}$ is

$$
\operatorname{pattern}\left(\mathrm{T}^{k}\right)=\left(\begin{array}{ccccccc}
\bullet & & \bullet & \bullet & & & \bullet \\
\bullet & & \bullet & \bullet & & & \bullet \\
\bullet & \bullet & \bullet & \bullet & \bullet & \bullet \\
& \bullet & & & \bullet & \bullet & \\
\bullet & & \bullet & \bullet & & & \bullet
\end{array}\right)
$$

and the two different types of rows are now

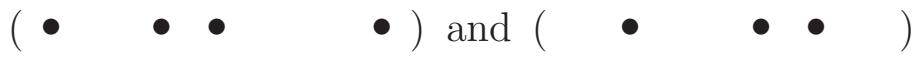

Consider now the case of two weakly coupled subsystems, see Fig. 2(b). The transfer matrix is 


$$
\mathbf{T}=\left(\begin{array}{cc}
\mathbf{T}_{\mathbf{1}} & \epsilon \mathbf{T}_{12} \\
\epsilon \mathbf{T}_{21} & \mathbf{T}_{2}
\end{array}\right)
$$

Where the factor $0<\epsilon<1$ in the off-diagonal blocks reflects the weak coupling. Power 2 of matrix $\mathbf{T}$ is

$$
\mathbf{T}^{2}=\left(\begin{array}{cc}
\mathbf{T}_{1}^{2}+\epsilon^{2} \mathbf{T}_{12} \mathbf{T}_{21} & \epsilon \mathbf{T}_{12,2} \\
\epsilon \mathbf{T}_{21,2} & \mathbf{T}_{2}^{2}+\epsilon^{2} \mathbf{T}_{21} \mathbf{T}_{12}
\end{array}\right) \simeq\left(\begin{array}{cc}
\mathbf{T}_{1}^{2} & \epsilon \mathbf{T}_{12,2} \\
\epsilon \mathbf{T}_{21,2} & \mathbf{T}_{2}^{2}
\end{array}\right)
$$

where $\mathbf{T}_{12,2}=\mathbf{T}_{1} \mathbf{T}_{12}+\mathbf{T}_{12} \mathbf{T}_{2}$ and $\mathbf{T}_{21,2}=\mathbf{T}_{21} \mathbf{T}_{1}+\mathbf{T}_{2} \mathbf{T}_{21}$. Note that only the leading term in the diagonal blocks is retained in the approximate expression of $\mathbf{T}^{2}$. It is straightforward to show that power $k$ is

$$
\mathbf{T}^{k}=\left(\begin{array}{cc}
\mathbf{T}_{1}^{k} & \epsilon \mathbf{T}_{12, k} \\
\epsilon \mathbf{T}_{21, k} & \mathbf{T}_{2}^{k}
\end{array}\right)
$$

with appropriate definitions of $\mathbf{T}_{12, k}$ and $\mathbf{T}_{21, k}$. Eq. (11) shows that the weak coupling between subsystems 1 and 2 is visible in $k$-order paths. For $k$ large enough, all four blocks in $\mathbf{T}^{k}$ are full matrices and the sparsity pattern of $\mathbf{T}^{k}$ is

$$
\text { pattern }\left(\mathrm{T}^{k}\right)=\left(\begin{array}{ccccccc}
\bullet & \bullet & \bullet & \bullet & 0 & 0 & 0 \\
\bullet & \bullet & \bullet & \bullet & 0 & 0 & 0 \\
\bullet & \bullet & \bullet & \bullet & 0 & 0 & 0 \\
0 & \bullet & \bullet & \bullet & 0 & 0 & 0 \\
0 & 0 & 0 & 0 & \bullet & \bullet & \bullet \\
0 & 0 & 0 & 0 & \bullet & \bullet & \bullet \\
1 & & & 0 & \bullet & \bullet
\end{array}\right)
$$

where the black dots $\bullet$ represent larger numerical values than the void dots $\circ$. A cluster analysis of the rows of $\mathbf{T}^{k}$ detects the different types of rows, irrespective of the node ordering. As the coupling between the two subsystems increases, the block structure of $\mathbf{T}^{k}$ becomes less clear. In the limit case of two strongly coupled subsystems, see Fig. 2 (c), they are regarded as a monolithic system.

Local vs global eigenmodes. Different definitions of coupling may be found in the literature [1, 22]. Some of them [23] are based on the nature of the eigenmodes: subsystems 1 and 2 are regarded as weakly coupled if the vibration eigenmodes are localised in subsystems 1 or 2, see Fig. 3(b), whereas they are considered to be strongly coupled if vibration eigenmodes are global, see Fig. 3(c). We show here that our approach based on the cluster analysis of the powers of $\mathbf{T}$ is consistent with this definition of coupling based in eigenmodes. Consider the basis $\left\{\mathbf{v}_{i}\right\}_{i=1}^{n}$ of eigenvectors of the transfer matrix $\mathbf{T}$, associated to eigenvalues $\left\{\lambda_{i}\right\}_{i=1}^{n}$,

$$
\mathbf{T v}_{i}=\lambda_{i} \mathbf{v}_{i} \quad i=1, \ldots, n
$$

and the corresponding eigendecomposition

$$
\mathbf{T}=\mathbf{V} \Lambda \mathbf{V}^{-1}
$$

where $\boldsymbol{\Lambda}$ is a diagonal matrix of eigenvalues and $\mathbf{V}$ is the matrix of eigenvectors:

$$
\boldsymbol{\Lambda}=\left(\begin{array}{cccc}
\lambda_{1} & & & \\
& \lambda_{2} & & \\
& & \ddots & \\
& & & \lambda_{n}
\end{array}\right) ; \quad \mathbf{V}=\left(\begin{array}{cccc}
\mid & \mid & \ldots & \mid \\
\mid & \mid & \ldots & \mid \\
\mathbf{v}_{1} & \mathbf{v}_{2} & \ldots & \mathbf{v}_{n} \\
\mid & \mid & \ldots & \mid
\end{array}\right)
$$




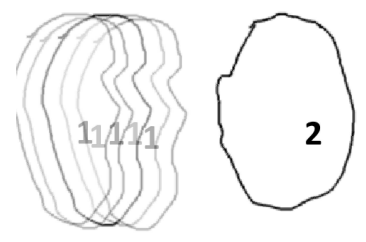

(1) (a)

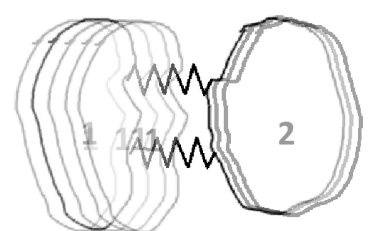

(b)

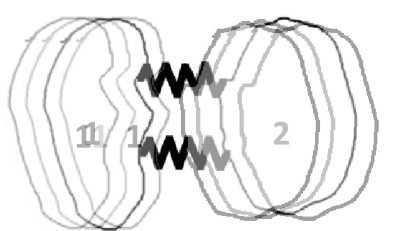

( / (c)

Figure 3: (a) Uncoupled subsystems modes; (b) Weakly coupled subsystems modes; (c) Strongly coupled subsystems modes

From Eq. (13), the powers of matrix $\mathbf{T}$ can be expressed as

$$
\mathbf{T}^{k}=\left(\mathbf{V} \boldsymbol{\Lambda} \mathbf{V}^{-1}\right)\left(\mathbf{V} \boldsymbol{\Lambda} \mathbf{V}^{-1}\right) \cdots\left(\mathbf{V} \boldsymbol{\Lambda} \mathbf{V}^{-1}\right)=\mathbf{V} \boldsymbol{\Lambda}^{k} \mathbf{V}^{-1}
$$

Eq. (13) and Eq. (15) show that any block structure that matrix $\mathbf{T}$ or $\mathbf{T}^{k}$ may have is associated to matrix $\mathbf{V}$, because matrix $\boldsymbol{\Lambda}$ is diagonal. By taking into account that the inverse of any (almost) block-diagonal matrix is another (almost) block-diagonal matrix, one can conclude that:

- For two uncoupled subsystems, the pattern of $\mathbf{V}$ is

$$
\operatorname{pattern}(\mathbf{V})=\left(\begin{array}{ccccccc}
\bullet & \bullet & \bullet & \bullet & & & \\
\bullet & \bullet & \bullet & \bullet & & & \\
\bullet & \bullet & \bullet & \bullet & & & \\
\bullet & \bullet & \bullet & \bullet & \bullet & \bullet & \bullet \\
& & & & \bullet & \bullet & \bullet \\
& & & \bullet & \bullet & \bullet
\end{array}\right)
$$

so eigenvectors of $\mathbf{T}$ are fully local.

- For two weakly coupled subsystems, the pattern of $\mathbf{V}$ is

$$
\operatorname{pattern}(\mathbf{V})=\left(\begin{array}{ccccccc}
\bullet & \bullet & \bullet & \bullet & 0 & 0 & 0 \\
\bullet & \bullet & \bullet & \bullet & 0 & 0 & 0 \\
\bullet & \bullet & \bullet & \bullet & 0 & 0 & 0 \\
\bullet & \bullet & \bullet & \bullet & 0 & 0 & 0 \\
0 & 0 & 0 & 0 & \bullet & \bullet & \bullet \\
0 & 0 & 0 & 0 & \bullet & \bullet & \bullet \\
0 & 0 & 0 & 0 & \bullet & \bullet & \bullet
\end{array}\right)
$$

so eigenvectors are localised, see Fig. 3(b).

- For two strongly coupled subsystems, matrix $\mathbf{V}$ does not have a clear block structure, so eigenvectors of $\mathbf{T}$ are global, see Fig. 3(c).

Note that the above discussion is based on the eigenvectors of $\mathbf{T}$, not on the eigenmodes of the mechanical system. However, as discussed in Appendix A, these two sets of vectors are closely related.

Contrast of the transfer matrix. Consider the transfer matrix for a system composed of two subsystems

$$
\mathbf{T}=\left(\begin{array}{cc}
\mathbf{T}_{1} & \alpha \mathbf{T}_{12} \\
\alpha \mathbf{T}_{21} & \mathbf{T}_{2}
\end{array}\right)
$$


with $\alpha=0$ for uncoupled subsystems $0<\alpha=\epsilon<1$ for weakly coupled subsystems and $\alpha \simeq 1$ for strongly coupled subsystems. We assume that the blocks $\mathbf{T}_{1}$ and $\mathbf{T}_{2}$ are invertible and define the contrast of $\mathbf{T}$ as

$$
\begin{aligned}
& \text { contrast }_{12}=\frac{1}{\left\|\mathbf{T}_{1}^{-1} \alpha \mathbf{T}_{12}\right\|}=\frac{1}{\alpha\left\|\mathbf{T}_{1}^{-1} \mathbf{T}_{12}\right\|} \\
& \text { contrast }_{21}=\frac{1}{\left\|\mathbf{T}_{2}^{-1} \alpha \mathbf{T}_{21}\right\|}=\frac{1}{\alpha\left\|\mathbf{T}_{2}^{-1} \mathbf{T}_{21}\right\|}
\end{aligned}
$$

The contrast provides a simple, scalar measure of the strength of the coupling between subsystems: the greater the contrast, the weaker the coupling. Note that the contrast of a matrix is invariant under scalar multiplication,

$$
\operatorname{contrast}(a \mathbf{T})=\operatorname{contrast}(\mathbf{T}) \quad \text { for any } \quad a \neq 0
$$

so it provides an scale-independent measure of the block diagonal dominance of a matrix. We examine now the contrast of matrix $\mathbf{T}^{k}$. Eq. (15) can be recast as

$$
\mathbf{T}^{k}=\mathbf{V} \Lambda^{k} \mathbf{V}^{-1}=\sum_{i=1}^{n} \lambda_{i}^{k} \mathbf{v}_{i} \mathbf{u}_{i}^{T} \approx \lambda_{n}^{k} \mathbf{v}_{n} \mathbf{u}_{n}^{T}
$$

where $\lambda_{i}$ and $\mathbf{v}_{i}$ are respectively the eigenvalues and the eigenvectors of $\mathbf{T}$, see Eq. (14), and $\mathbf{u}_{i}^{T}$ are rows of the matrix $\mathbf{V}^{-1}$. Note that $\mathbf{T}$ may be approximated, for $k$ large enough, by the term associated to the dominant eigenvalue $\lambda_{n}$.

From Eqs. (21) and (22), it follows that

$$
\operatorname{contrast}\left(\mathbf{T}^{k}\right) \approx \operatorname{contrast}\left(\lambda_{n}^{k} \mathbf{v}_{n} \mathbf{u}_{n}^{T}\right)=\operatorname{contrast}\left(\mathbf{v}_{n} \mathbf{u}_{n}^{T}\right)
$$

Equation (23) shows that irrespectively of the dominant eigenvalue $\lambda_{n}$, the contrast of $\mathbf{T}^{k}$ tends to a limit when $k$ growths. From a practical point of view, this means that there is not need to fine-tune the value of $k$ : any value large enough may be used. Furthermore, even if $\mathbf{T}^{k}$ tends to $\infty$ when its norm is larger than one, the contrast will not: the method is stable.

\subsection{Estimation of the coupling strength}

An important aspect is to quantify the degree of coupling between subsystems. In other words, the correctness of the solution obtained if a subsystem is considered as isolated from the other parts.

Several definitions of the weak coupling between subsystems exist, see for example $[1,22]$. Consider a mechanical system described as

$$
\left(\begin{array}{l}
V_{1} \\
V_{2}
\end{array}\right)=\left(\begin{array}{ll}
Y_{11} & Y_{12} \\
Y_{21} & Y_{22}
\end{array}\right)\left(\begin{array}{l}
F_{1} \\
F_{2}
\end{array}\right)
$$

where $Y$ are the admittances, $V$ the velocities and $F$ the forces, and the admittance integrals defined by integration of the whole frequency range

$$
I_{j k} \equiv \int_{-\infty}^{\infty}\left|Y_{j k}\right|^{2} d \omega
$$


Ungar [22] defines that two systems are weakly coupled if the transfer admittance integrals are equal, $I_{12}=I_{21}$, and their values are smaller than those of the selfadmittance integrals $I_{11}$ and $I_{22}$. Moreover, the transfer impedances and admittances $Z_{12}, Z_{21}, Y_{12}, Y_{21}$, must not affect the values of $I_{11}$ and $I_{22}$ significantly. That definition is provided in terms of the energy and integrated in the frequency domain. This is less restrictive that conditions that must be satisfied at each frequency.

An estimation of the coupling between subsystems can also be done through the analysis of the largest eigenvalue of a coupling matrix [24]. The matrix representation of the mechanical system must be reorganised as proposed in [25] which allows a separation of the coupling part. A similar block matrix structure, combined with the path concept, is considered here in order to define the coupling strength between subsystems. We consider a linear system of equations partitioned in coupled blocks

$$
\left(\begin{array}{ll}
\mathbf{A}_{11} & \mathbf{A}_{12} \\
\mathbf{A}_{21} & \mathbf{A}_{22}
\end{array}\right)\left(\begin{array}{l}
\mathbf{x}_{1} \\
\mathbf{x}_{2}
\end{array}\right)=\left(\begin{array}{l}
\mathbf{b}_{1} \\
\mathbf{b}_{2}
\end{array}\right)
$$

where $\mathbf{A}_{11}$ and $\mathbf{A}_{22}$ are the dynamical matrices of two sets of degrees of freedom and $\mathbf{A}_{12}$ and $\mathbf{A}_{21}$ their coupling matrices. Our purpose is to establish which is the difference between the fully coupled solution and the solution of the uncoupled system

$$
\left(\begin{array}{cc}
\mathbf{A}_{11} & \mathbf{0} \\
\mathbf{0} & \mathbf{A}_{22}
\end{array}\right)\left(\begin{array}{l}
\mathbf{x}_{1}^{\prime} \\
\mathbf{x}_{2}^{\prime}
\end{array}\right)=\left(\begin{array}{l}
\mathbf{b}_{1} \\
\mathbf{b}_{2}
\end{array}\right)
$$

The linear system in (26) can be rewritten in terms of transfer matrices

$$
\left(\begin{array}{l}
\mathbf{x}_{1} \\
\mathbf{x}_{2}
\end{array}\right)=\left(\begin{array}{cc}
\mathbf{A}_{11} & \mathbf{0} \\
\mathbf{0} & \mathbf{A}_{22}
\end{array}\right)^{-1}\left(\begin{array}{l}
\mathbf{b}_{1} \\
\mathbf{b}_{2}
\end{array}\right)-\left(\begin{array}{cc}
\mathbf{0} & \mathbf{A}_{11}^{-1} \mathbf{A}_{12} \\
\mathbf{A}_{22}^{-1} \mathbf{A}_{21} & \mathbf{0}
\end{array}\right)\left(\begin{array}{l}
\mathbf{x}_{1} \\
\mathbf{x}_{2}
\end{array}\right)
$$

which is useful in order to relate the difference between the coupled solution in the subsystem 1, $\mathbf{x}_{1}$ and the uncoupled solution $\mathbf{x}_{1}^{\prime}$

$$
\mathbf{x}_{1}=\mathbf{x}_{1}^{\prime}-\mathbf{A}_{11}^{-1} \mathbf{A}_{12} \mathbf{x}_{2}
$$

If the force is applied on subsystem 1 and there is no local resonance on subsystem 2 , $\left\|\mathbf{x}_{1}\right\|>\left\|\mathbf{x}_{2}\right\|$ and then

$$
\frac{\left\|\mathbf{x}_{1}-\mathbf{x}_{1}^{\prime}\right\|}{\left\|\mathbf{x}_{1}\right\|} \leq\left\|\mathbf{A}_{11}^{-1} \mathbf{A}_{12}\right\|=\frac{1}{\text { contrast }_{12}}
$$

It is clear from Eq. (30) that the contrast of matrix $\mathbf{A}$ is a measure of the coupling between subsystems 1 and 2: the greater the contrast, the more accurate the isolated solution.

\section{Numerical examples and analysis}

The ideas exposed in Section 2 are illustrated by means of mechanical and acoustic problems solved in the frequency domain (steady harmonic). 


\subsection{Example 1: Two plates connected by means of five (soft) springs}

The first example consists of two plates of different size and linked through five springs, see Fig. 4. The geometric and mechanical properties of the plates are listed in Table 1 and the stiffness of the springs and their positions are summarized in Table 2 (' $\mathrm{x}$ ' and ' $y$ ' coordinate axes shown in Fig. 7). The results are shown for an excitation frequency of $100 \mathrm{~Hz}$. The system matrix $\mathbf{A}$ is obtained with finite differences, using a regular grid (spacing $0.1 \mathrm{~m}$ ) in both plates.

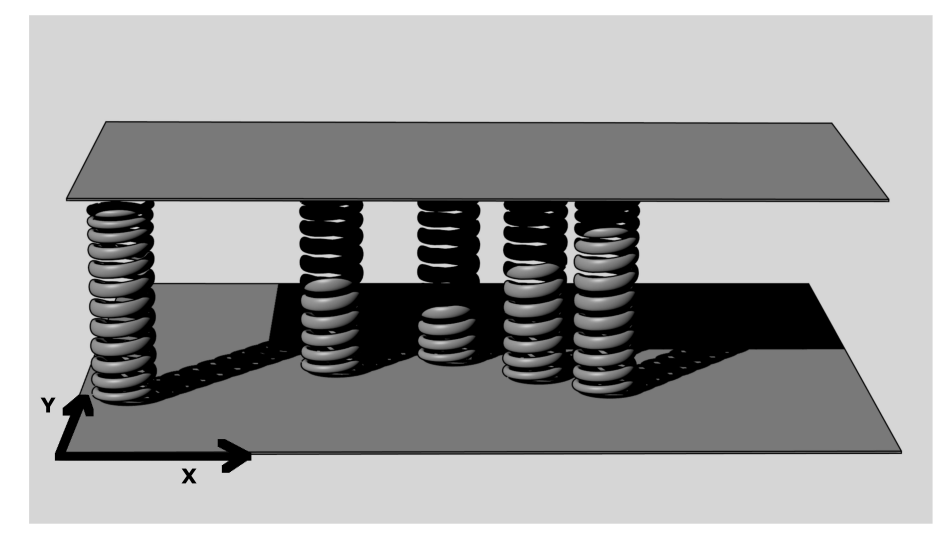

Figure 4: Two plates and five springs as described in Table 2. Computation at 100 $\mathrm{Hz}$.

\begin{tabular}{ccccccc}
\hline Material & $\rho_{v}\left(\mathrm{~kg} / \mathrm{m}^{3}\right)$ & $\nu$ & $E(\mathrm{~Pa})$ & $L_{x}(\mathrm{~m})$ & $L_{y}(\mathrm{~m})$ & $h(\mathrm{~m})$ \\
\hline Concrete & 2000 & 0.2 & $2 \cdot 10^{10}$ & 2 & 2 & 0.03 \\
Concrete & 2000 & 0.2 & $2 \cdot 10^{10}$ & 1 & 2 & 0.03 \\
\hline
\end{tabular}

Table 1: Geometric and mechanical properties of the two plates $\left(\rho_{v}\right.$ is the volumetric density, $\nu$ is the Poisson's ratio, $E$ is the Young modulus, $L_{x}, L_{y}$ the plate dimensions and $h$ the plate thickness).

\begin{tabular}{lccc}
\hline Spring & $K(\mathrm{~N} / \mathrm{m})$ & $x(\mathrm{~m})$ & $y(\mathrm{~m})$ \\
\hline 1 & $2 \cdot 10^{5}$ & 0.3 & 0.4 \\
2 & $2 \cdot 10^{5}$ & 0.6 & 0.1 \\
3 & $2 \cdot 10^{5}$ & 0.2 & 0.4 \\
4 & $2 \cdot 10^{5}$ & 0.5 & 0.5 \\
5 & $2 \cdot 10^{5}$ & 0.1 & 0.1 \\
\hline
\end{tabular}

Table 2: Stiffness $K$ and position of each spring that connects the plates.

Fig. 5 shows the sparsity pattern of the transfer matrix of the system T. Note that a natural nodal ordering is used (first the nodes in the bottom plate, then the 
nodes in the top plate). This facilitates the interpretation of results, but, as discussed in Section 2.3.2, is not required by the clustering algorithm.

Fig. 6 shows power $k=21$ of the transfer matrix that clearly presents a block structure. The coefficients of the diagonal blocks are representative of paths that start and end at the same plate. On the contrary, the coefficients in the out-ofdiagonal or coupling blocks represent paths that begin and end in different plates. In this case the nodal ordering helps to see the block structure.

Fig. 7 illustrates the clustering process. The dendrogram identifies two clusters of nodes, coloured in red and blue, that correspond to the two plates. Note that the lower part of the dendrogram is barely visible, because the correlation distance between nodes in the same plate is much smaller than the correlation distance between the two plates.

We have checked that the vibration fields in plate 1 subject to random forces are virtually identical for the coupled and uncoupled cases. This is a consequence of the large contrast of $1.1 \cdot 10^{3}$.

We can conclude that the method allows us to identify the subsystems in agreement with the concept that a subsystem is a set of nodes with similar behaviour.

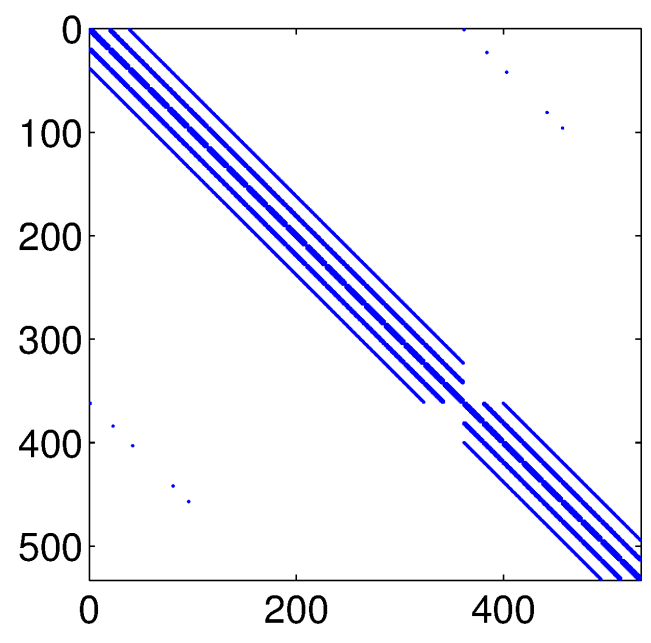

Figure 5: Sparsity pattern of the transfer matrix of the example with two plates and five springs as described in Table 2.

\subsection{Example 2: Four plates connected by springs}

The second example consists of four plates of equal size $(1 \mathrm{~m} \times 2 \mathrm{~m})$ and linked by means of springs, see Fig. 8.

The positions of the springs in the plates are the same as in Section 3.1 and the spring properties are given in Table 4. The springs linking plates 2 and 3 are more flexible than the ones linking plates 1 and 2 and plates 3 and 4 . With this configuration we expect to find two subsystems composed of two plates each.

Fig. 9 shows the transfer matrix $\mathbf{T}$ of the four plates. Fig. 10(a) shows $\mathbf{T}^{k}$, with $k=28$. A $4 \times 4$ block structure can be clearly seen. Moreover the colour plot allows the 


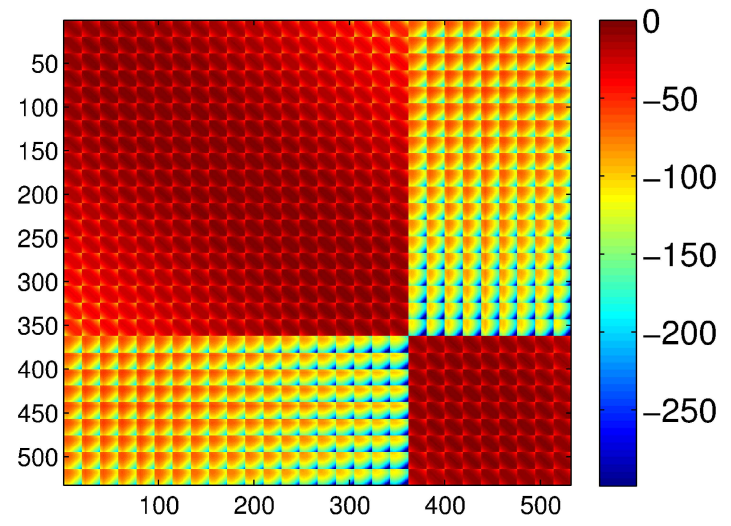

Figure 6: Block structure of matrix $\mathbf{T}^{21}$. The colour represents the normalised entries in the $\mathrm{dB}$ scale, $10 \log _{10}\left(\left|t_{i j}^{21}\right| / \max \left(\left|t_{i j}^{21}\right|\right)\right)$.
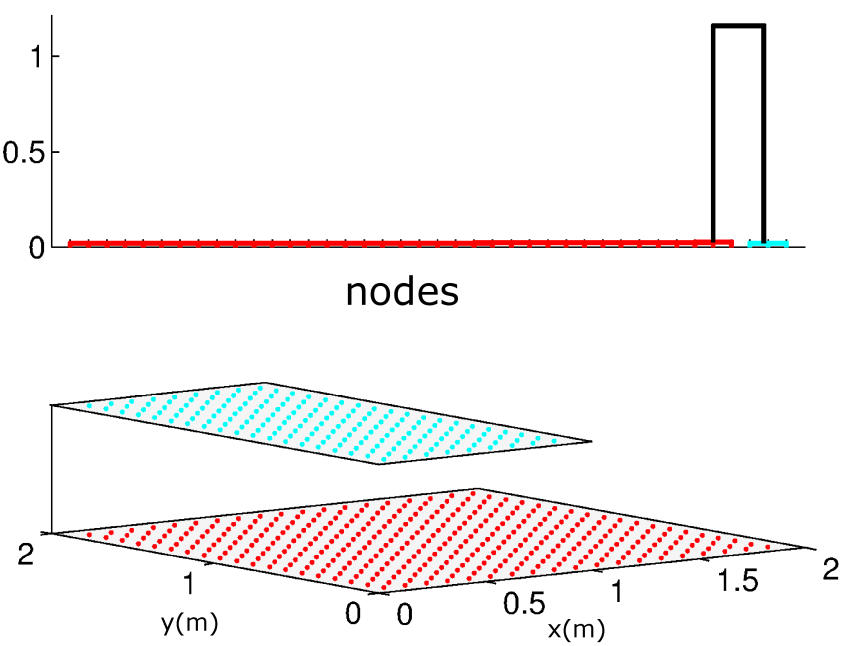

Figure 7: Top: Dendrogram obtained with $\mathbf{T}^{21}$. Bottom: Dendrogram nodes assigned to the plates.

\begin{tabular}{ccccccc}
\hline Material & $\rho_{v}\left(\mathrm{~kg} / \mathrm{m}^{3}\right)$ & $\nu$ & $E(\mathrm{~Pa})$ & $L_{x}(\mathrm{~m})$ & $L_{y}(\mathrm{~m})$ & $h(\mathrm{~m})$ \\
\hline Concrete & 2000 & 0.2 & $2 \cdot 10^{10}$ & 1 & 2 & 0.03 \\
\hline
\end{tabular}

Table 3: Mechanical properties of the four plates linked by means of springs.

\begin{tabular}{llccc}
\hline Plates & Spring & $K(\mathrm{~N} / \mathrm{m})$ & $x(\mathrm{~m})$ & $y(\mathrm{~m})$ \\
\hline $1-2$ & $1-5$ & $2 \cdot 10^{10}$ & As in example 1 & As in example 1 \\
$2-3$ & $6-10$ & $2 \cdot 10^{5}$ & As in example 1 & As in example 1 \\
$3-4$ & $11-15$ & $2 \cdot 10^{10}$ & As in example 1 & As in example 1 \\
\hline
\end{tabular}

Table 4: Properties of the five springs that link contiguous plates. 


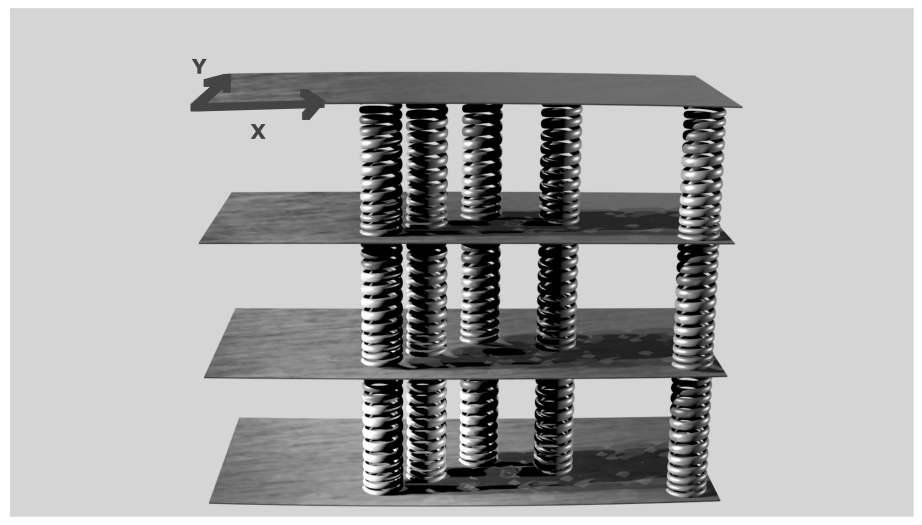

Figure 8: Four plates connected by springs as described in Table 4. Computation at $100 \mathrm{~Hz}$.

visual identification of two mechanical subsystems (plates 1-2 and plates $3-4$ ). Two different degrees of coupling exist. On the one hand a clearly weak coupling between subsystems. This is caused by the flexible springs that link plates 2 and 3 . On the other hand we can see that inside each subsystem there is a much stronger coupling between the two plates. This causes the cluster analysis to define two subsystems instead of four. The two subsystems are also clearly visible in matrix $\mathbf{V}$ of eigenvectors of the direct transfer matrix T, see Fig. 10(b).

Fig. 11 shows the dendrogram of the cluster analysis. It is clear regarding the length of the branches that it suggests the definition of only two subsystems instead of four.

A comparison of the solutions obtained by solving the whole problem (four plates) or an isolated subsystem (two plates) is made. A measure of the difference is

$$
e=\frac{\left\|\mathbf{u}_{\text {iso }}-\mathbf{u}_{\text {all }}\right\|}{\left\|\mathbf{u}_{\text {all }}\right\|}
$$

where $\|\bullet\|$ is the Euclidean norm, $\mathbf{u}_{\text {iso }}$ is the vector of nodal displacements in the isolated solution and $\mathbf{u}_{\text {all }}$ is the vector of nodal displacements in the coupled solution.

If the subsystem formed by plates 1 and 2 is analysed isolatedly, a very low error of $e=8.7 \cdot 10^{-7}$ with respect to the coupled solution is obtained. This is related to the very high contrast of 147 .

If the subsystems identified by the cluster analysis are not respected and plate 1 is analysed isolatedly, a large error of $e=0.6$ is committed. This is explained by the low contrast of 0.21 .

Fig. 12 shows the evolution of the contrast of $\mathbf{T}^{k}$ with respect to power $k$. Note that the contrast between plates 2 and 3, linked by soft springs, is much larger than the contrast between plates 1 and 2 or plates 3 and 4 , linked by much stiffer springs. Fig. 12 also shows that the contrasts stabilise as $k$ increases, to values that can be accurately estimated from the dominant eigenvectors, see Eq. (23). 


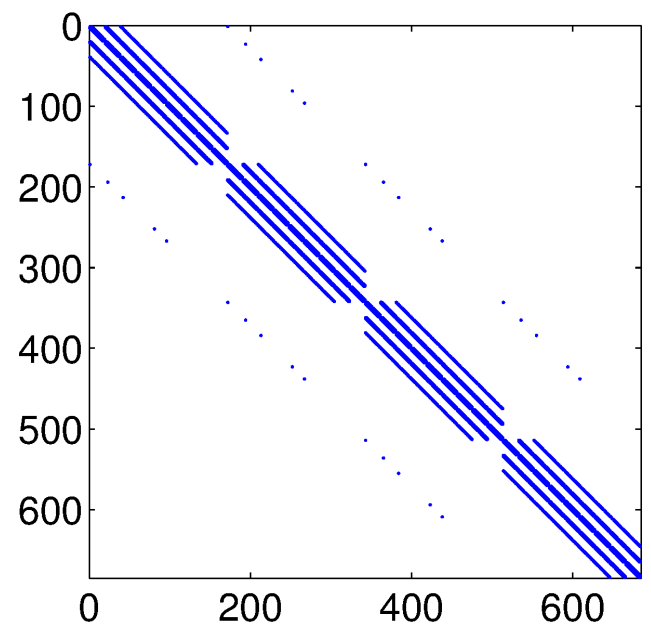

Figure 9: Sparsity pattern of the transfer matrix. Four plates connected by springs as described in Table 4.

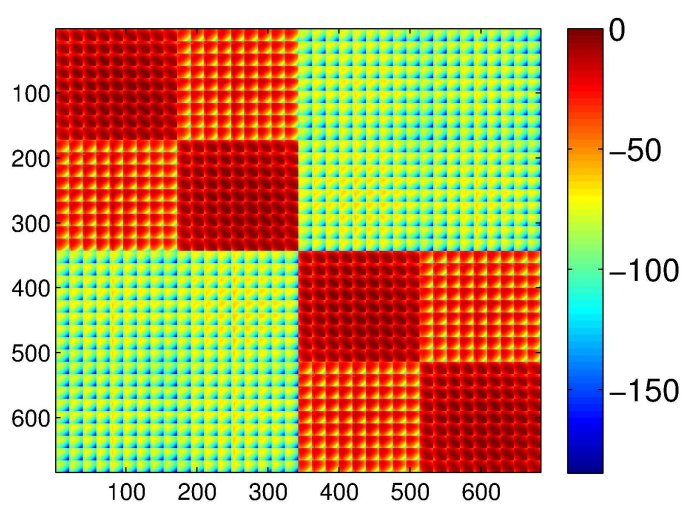

(a)

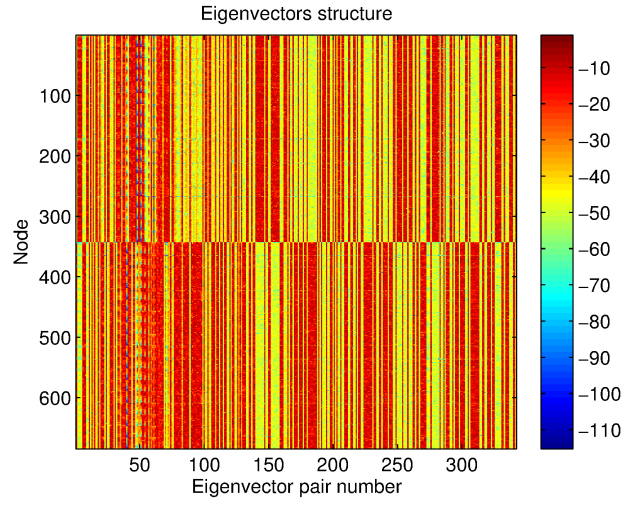

(b)

Figure 10: (a) Block structure of matrix $\mathbf{T}^{28}$. The colour represents the normalised entries in the $\mathrm{dB}$ scale; (b) matrix $\mathbf{V}$ of eigenvectors of the direct transfer matrix.

\subsection{Two acoustically coupled rooms}

The clustering method is applied to a two-dimensional acoustic problem: two rectangular rooms connected through a one-dimensional hole, see Fig. 13. The room sizes are $20 u \times 23 u$ and $25 u \times 23 u$ ( $u$ is a unit length). Two different situations are considered. In the first one, the hole length is $5 u$, and the acoustic wavelength (ratio between the speed of sound and frequency, $\lambda_{a c}=c / f$ ) satisfies $\lambda_{a c}=1.26 \times 5 u$. This is the case of hole size which is similar to the acoustic wavelength. In the second one, the hole length is $1 u$, and the acoustic wavelength satisfies $\lambda_{a c}=76.3 \times 1 u$. This is the case of hole size very different to the acoustic wavelength. The system matrix $\mathbf{A}$ is obtained by discretising the Helmholtz equation by means of finite volume-based technique.

The degree of coupling between the two rooms is controlled by the ratio of the 

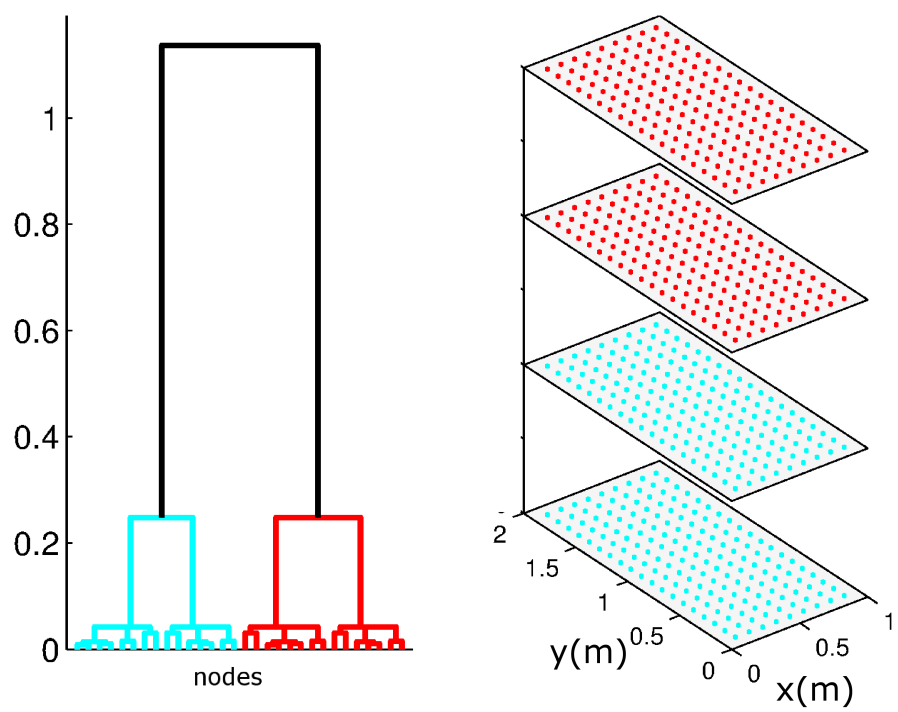

Figure 11: Left: Dendrogram obtained with $\mathbf{T}^{28}$. Right: Dendrogram nodes assigned to the plates

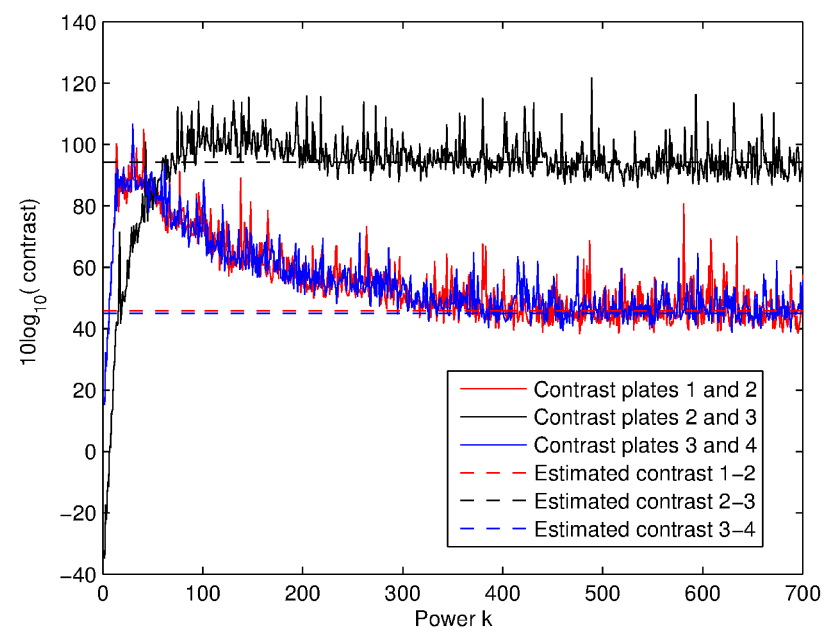

Figure 12: Evolution of contrast of matrix $\mathbf{T}^{k}$ with power $k$ (solid lines) vs value estimated according to Eq. (23) (dashed lines).

wavelength to the hole size. Two different cases are shown here with ratios 1.26 and 76.3 respectively. Weak coupling is only expected in the second case due to the large difference between the acoustic wavelength and the hole width.

The outputs of the clustering process for the case the small ratio are shown in Figs. 14 and 15. In that case, the hole size is very similar to the acoustic wavelength. As expected, the dendrogram in Fig. 15 identifies only one system. The interaction between both rooms is very important due to the large width of the hole with respect to the wavelength.

If the rooms are analysed isolatedly, the error computed according to Eq. (31) 


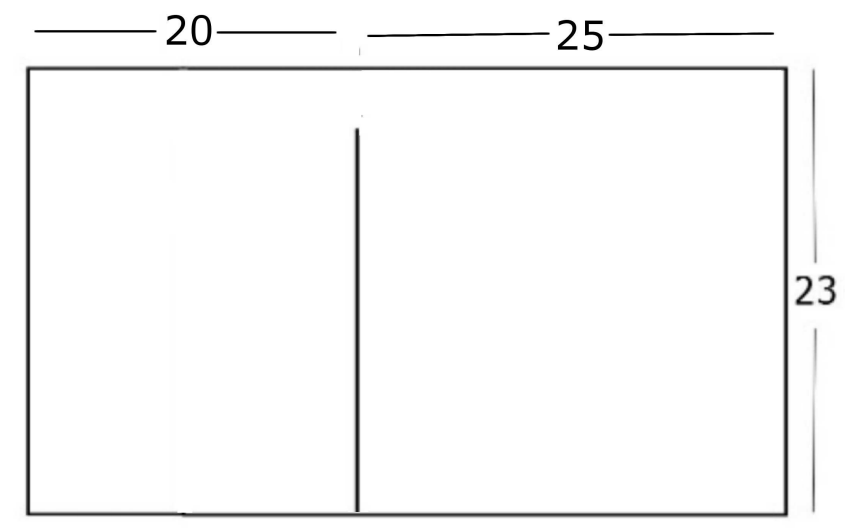

Figure 13: Sketch of the two rectangular rooms connected by means of a hole. The room sizes are $20 u \times 23 u$ and $25 u \times 23 u$ ( $u$ is a unit length). Two different holes have been considered: $1 u$ and $5 u$ in length.

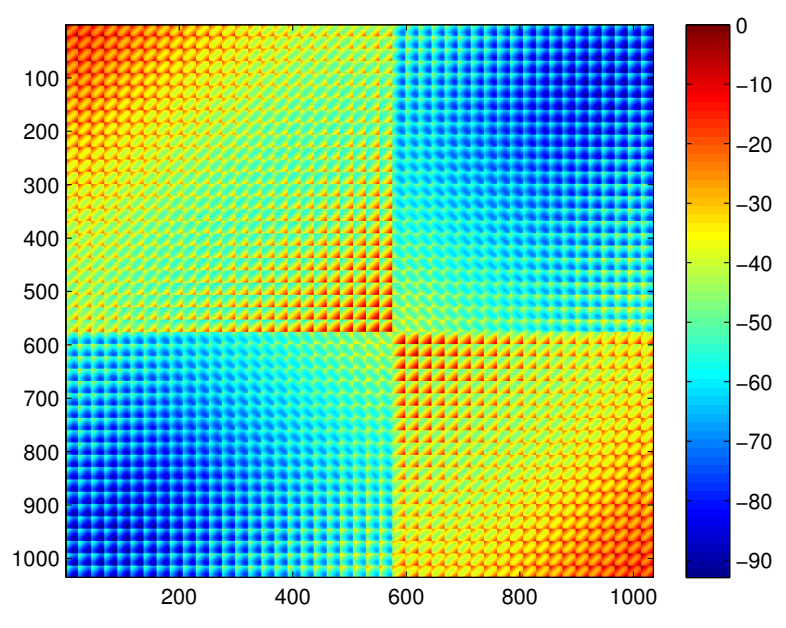

Figure 14: Block structure of matrix $\mathbf{T}^{343}$. The colour represents the normalised entries in the $\mathrm{dB}$ scale. Wavelength to hole size ratio: 1.26.

is $208 \%$ for the left room and $191 \%$ for the right room, with contrasts $4.8 \cdot 10^{-2}$ and $2.8 \cdot 10^{-2}$ respectively. It is clear that considering both rooms as independent subsystems is not a valid option.

Figs. 16 and 17 show the outputs of the clustering process for the large ratio of wavelength to hole size: 76.3. Now, both rooms are identified as almost independent subsystems. This can be concluded by the aspect of the dendrogram and is corroborated by the error measure. In that case, Eq. (31) provides values of $9 \%$ for the left room and $11 \%$ for the right one, which were expected due to the smaller value of the contrasts ( 0.27 for both). 

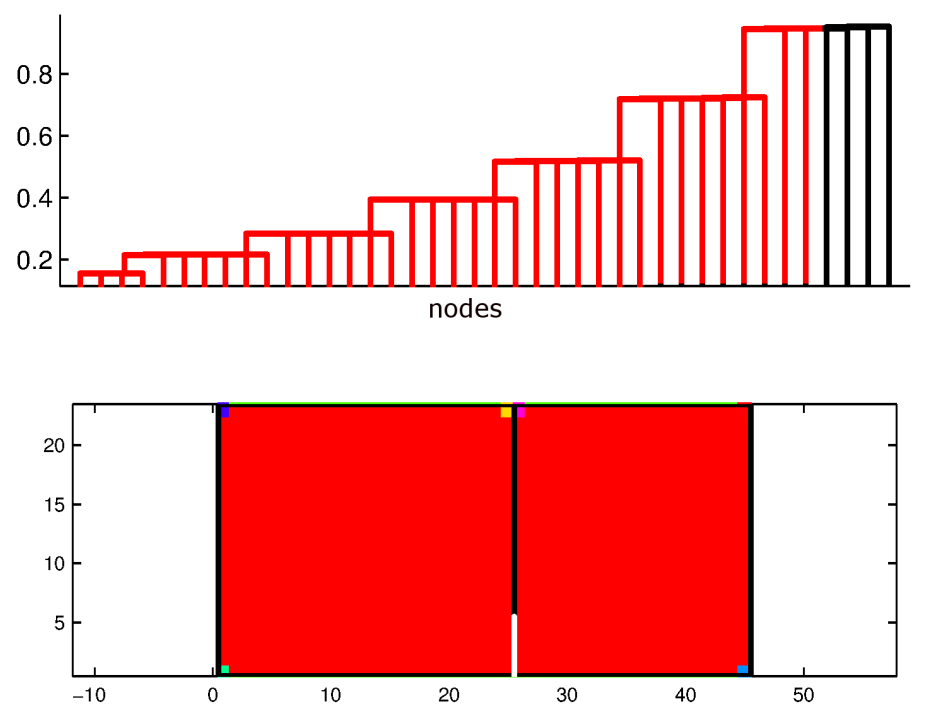

Figure 15: Top: Dendrogram . Wavelength to hole size ratio: 1.26; Bottom: Identified groups of nodes assigned to the room.

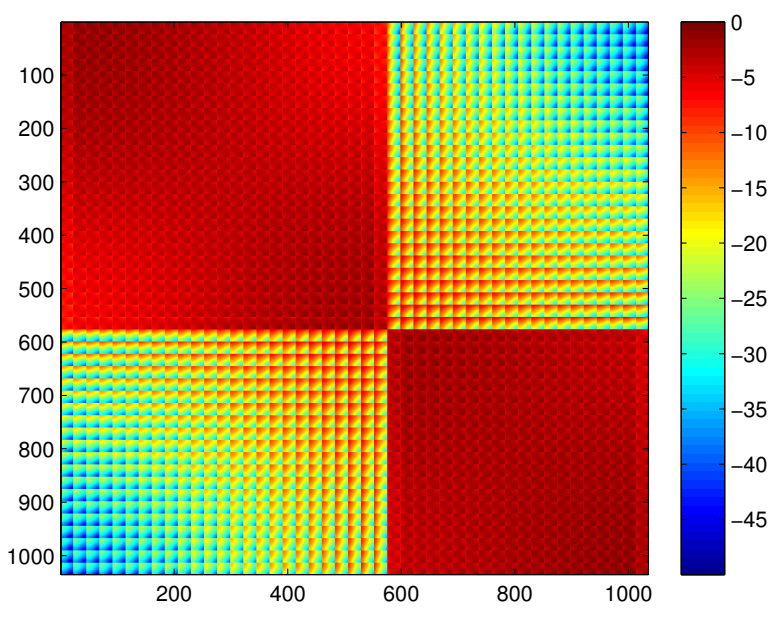

Figure 16: Block structure of matrix $\mathbf{T}^{343}$. The colour represents the normalised entries in the $\mathrm{dB}$ scale. Wavelength to hole size ratio: 76.3.

\subsection{Degrees of freedom selection: problem size reduction}

In the previous examples, the transfer matrix is directly obtained from the dynamical matrix of the system according to Eq. (4). The dynamical matrix was generated by means of numerical techniques. The number of unknowns is quite large because the discretisation of a mechanical or acoustic system must be done according to physical criteria. Enough nodes must be considered in order to properly describe the displacement or acoustic waves. Consequently, the transfer matrix $\mathbf{T}$ is a large matrix.

This has two important implications. On the one hand, when applying the pro- 

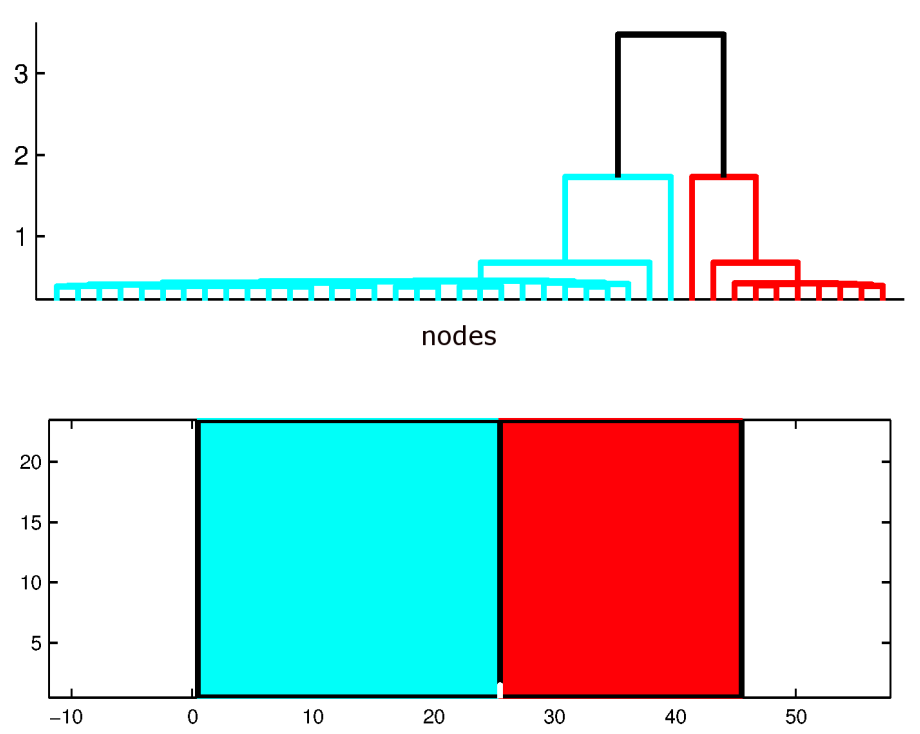

Figure 17: Top: Dendrogram. Wavelength to hole size ratio: 76.3; Bottom: Identified groups of nodes assigned to the room.

posed substructuring method, the cluster analysis has to deal with a large amount of data. This makes the process slow. On the other hand, a large power $k$ of matrix $\mathbf{T}$ must be considered. $\mathbf{T}^{k}$ is representative of the $k$-order paths. First-order paths in the numerical techniques are very short, because they link contiguous nodes. The clustering method requires paths that explore all the problem geometry; hence, a large value of $k$ is required.

What it is proposed here is to consider a more elaborated (post-processed) transfer matrix. This accounts only for some strategically chosen nodes on the system. They can be uniformly distributed all around the geometry or intuitively chosen as it would be done in a laboratory experiment where it is needed to choose a reduced number of measuring points (this is often limited by the number of available measuring channels).

The coefficients of the transfer matrix $\mathbf{T}$ are now computed by means of the solution of several problems as

$$
t_{i j}=\frac{s_{j}}{s_{i}}
$$

where $s_{j}$ is the signal at node $j$ when a unit excitation is applied at node $i$ and the signal of all the nodes $r \neq i, j$ is blocked. Note that for each coefficient $t_{i j}$ a problem has to be solved. The whole procedure is detailed in [19, 20].

This transfer matrix with less degrees of freedom has an additional advantage with respect to the one computed by means of Eq. (4). It is a full matrix with direct connectivity between all the selected nodes. This drastically reduces the power $k$ of matrix $\mathbf{T}$ required to represent paths that cover the whole geometry.

To illustrate this approach, the problem with four plates of Section 3.2 is revisited here. The only difference is that now all the springs have the same stiffness of 2 . $10^{7} \mathrm{~N} / \mathrm{m}$. The discretisation of the four plates leads to a system with 684 degrees of freedom. This is later reduced to 48 degrees of freedom, 12 points per plate. 
The clusters can be clearly identified from matrix $\mathbf{T}$ itself, with no need of computing powers. This is shown in Figs. 18 and 19, which show that each plate is identified as one subsystem. These smaller transfer matrices are the ones typically obtained in a laboratory or in situ measurement.

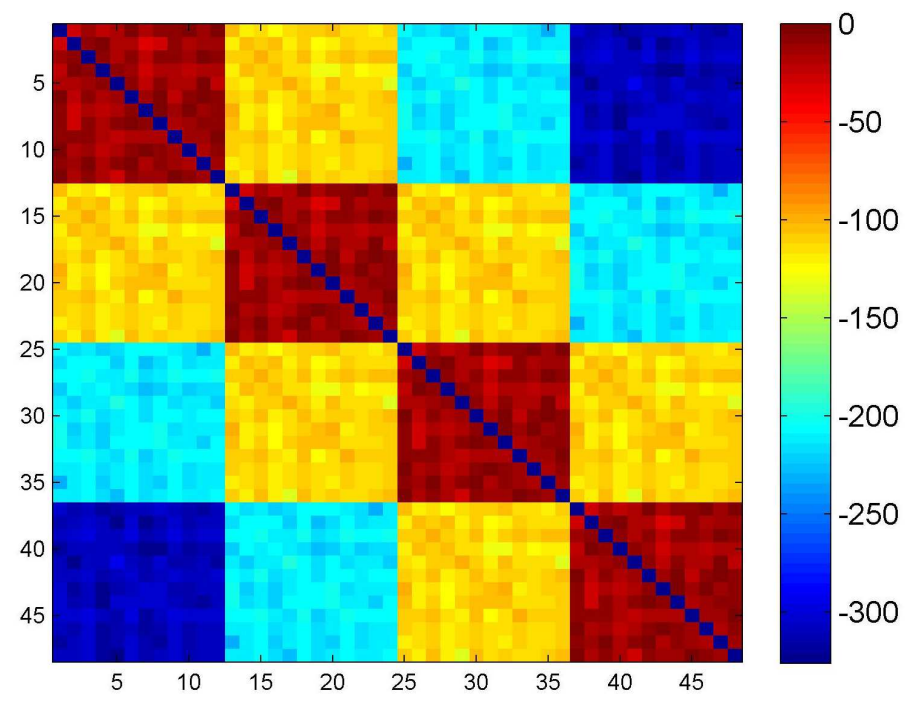

Figure 18: Block structure of matrix $\mathbf{T}$. The colour represents the normalised entries in the $\mathrm{dB}$ scale, $10 \log _{10}\left(\left|a_{i j}\right| / \max \left(\left|a_{i j}\right|\right)\right)$.

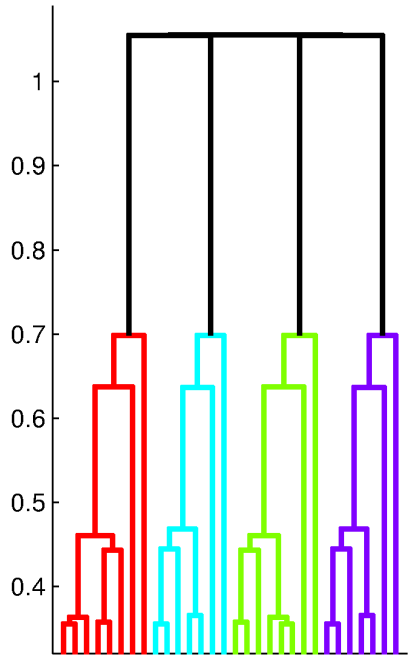

nodes

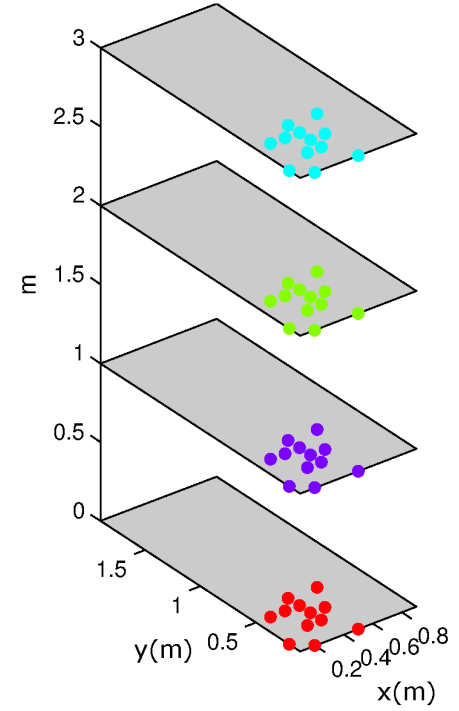

Figure 19: Left: Dendrogram obtained with $\mathbf{T}^{1}$. Right: Grouping of the 12 randomly selected nodes on each of the four plates (one subsystem per plate in this example). 


\section{Conclusions}

The main conclusions of the research are as follows:

1. A methodology for the automatic identification of subsystems has been proposed. It is based on the cluster analysis applied to powers of the transfer matrix. It is shown, through numerical examples, how the method works and is able to identify subsystems on its own without additional information or guidelines.

2. The subsystems are more clearly identified when higher powers of the transfer matrix are considered. This agrees with the explanations of Section 2.3.2.

3. If the transfer matrix represents all the degrees of freedom in a numerical discretisation of the system, the power required in order to properly identify the subsystems can be quite high. This can be linked with the representation of paths. They must be large enough in order to cover all the domain. On the contrary, if the transfer matrix is manipulated in order to directly represent long paths, as described in Section 3.4, a lower powers is required. This second option makes the method more efficient for the characterisation of large systems.

4. An important parameter in order to explain the good behaviour of the proposed technique is the contrast of the matrix. It is also used as an estimator of the coupling strength between subsystems.

5. A fast method to estimate the contrast of $\mathbf{T}^{k}$ is proposed. It is based on the dominant eigenvalue of the transfer matrix $\mathbf{T}$ and the associated eigenvector(s).

\section{A Eigenvectors of T vs. vibration eigenmodes}

Consider a mechanical system with stiffness matrix $\mathbf{K}$ and mass matrix $\mathbf{M}$. The eigenmodes $\boldsymbol{\Phi}_{i}$ and eigenfrequencies $\omega_{i}$ verify

$$
\mathbf{K} \Phi_{i}=\omega_{i}^{2} \mathbf{M} \Phi_{i} \quad i=1 \ldots n
$$

If this mechanical system is subject to an harmonic excitation of frequency $\omega_{e x c}$ and phasor $\mathbf{f}$, the phasor $\mathbf{u}$ of displacements is the solution of

$$
\mathbf{A}\left(\omega_{e x c}\right) \mathbf{u}=\mathbf{f}
$$

with

$$
\mathbf{A}\left(\omega_{\text {exc }}\right)=\mathbf{K}-\omega^{2} \mathbf{M}
$$

We are interested in exploring the relation between the eigenmodes $\boldsymbol{\Phi}_{i}$ and the eigenvectors $\mathbf{v}_{i}$ of the transfer matrix $\mathbf{T}\left(\omega_{\text {exc }}\right)$ associated to the linear system (34). We start by assuming that the mass matrix is diagonal (i.e. all system mass concentrated at nodes) and that all diagonal entries of $\mathbf{M}$ and $\mathbf{K}$ are equal:

$$
\mathbf{M}=m_{0} \mathbf{I}
$$




$$
\mathbf{K}=k_{0} \mathbf{I}+\mathbf{L}+\mathbf{U}
$$

This result in the system matrix

$$
\mathbf{A}\left(\omega_{e x c}\right)=\left(k_{0}-\omega_{e x c}^{2} m_{0}\right) \mathbf{I}+\mathbf{L}+\mathbf{U}
$$

and the transfer matrix

$$
\mathbf{T}\left(\omega_{e x c}\right)=-\frac{1}{k_{0}-\omega_{e x c}^{2} m_{0}}(\mathbf{L}+\mathbf{U})
$$

Under these conditions, the vibration eigenmodes $\boldsymbol{\Phi}_{i}$ are equal to the eigenvectors of $\mathbf{T}\left(\omega_{\text {exc }}\right)$ for any excitation frequency $\omega_{\text {exc }} \neq \omega_{0}=\sqrt{\frac{k_{0}}{m_{0}}}$. Indeed, by combining equations (33), (36), (37) and (39), one obtains

$$
\begin{aligned}
\mathbf{T}\left(\omega_{e x c}\right) \boldsymbol{\Phi}_{i} & =-\frac{1}{k_{0}-\omega_{e x c}^{2} m_{0}}(\mathbf{L}+\mathbf{U}) \boldsymbol{\Phi}_{i}=-\frac{1}{k_{0}-\omega_{e x c}^{2} m_{0}}\left(\mathbf{K}-k_{0} \mathbf{I}\right) \boldsymbol{\Phi}_{i} \\
& =\frac{\omega_{0}^{2}-\omega_{i}^{2}}{\omega_{0}^{2}-\omega_{e x c}^{2}} \boldsymbol{\Phi}_{i}=\lambda_{i}\left(\omega_{e x c}\right) \boldsymbol{\Phi}_{i}
\end{aligned}
$$

Eq. (40) also shows that, contrary to the eigenvectors $\mathbf{v}_{i} \equiv \boldsymbol{\Phi}_{i}$, the eigenvalues $\lambda_{i}$ do depend on the excitation frequency.

Without the simplifying assumptions Eq. (36) and Eq. (37), the vibration eigenmodes are not equivalent to the eigenvectors of $\mathbf{T}$. However, there is still a qualitative relation. Weakly coupled subsystems results in small off-diagonal blocks in the stiffness matrix $\mathbf{K}$ that lead to local vibration eigenmodes; they also result in small off-diagonal blocks in the system matrix $\mathbf{A}\left(\omega_{\text {exc }}\right)$ and in the transfer matrix $\mathbf{T}\left(\omega_{\text {exc }}\right)$ that lead to local eigenvectors.

An example can be seen in the Fig. 20. Some eigenvectors of $\mathbf{T}$ are compared with the corresponding eigenmodes of a room $12 \mathrm{~m}$ wide and $14 \mathrm{~m}$ long.

\section{Acknowledgements}

LaCàN research group is grateful for the sponsorship/funding received from Generalitat de Catalunya (Grant number 2014-SGR-1471). The authors are grateful to Ester Cierco who collaborated in the companion video production process as voice in off. 


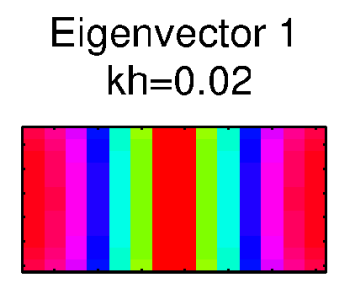

Eigenvector 1 $\mathrm{kh}=0.3$

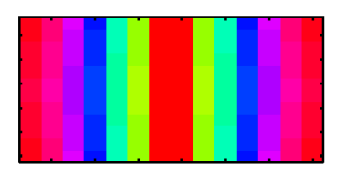

Eigenmode 1,0

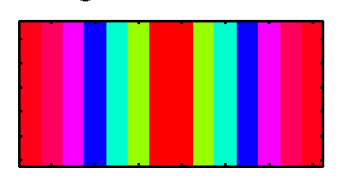

Eigenvector 2

$\mathrm{kh}=0.02$

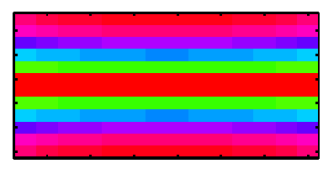

Eigenvector 2

$\mathrm{kh}=0.3$

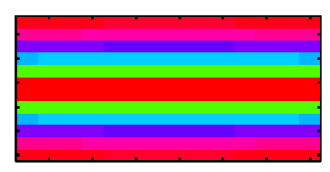

Eigenmode 0,1

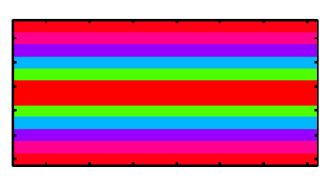

Eigenvector 3

$\mathrm{kh}=0.02$

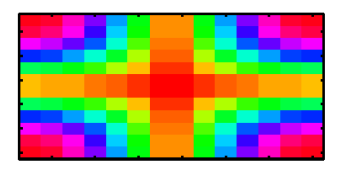

Eigenvector 3

$\mathrm{kh}=0.3$

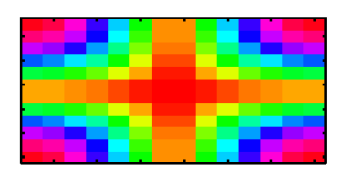

Eigenmode 1,1

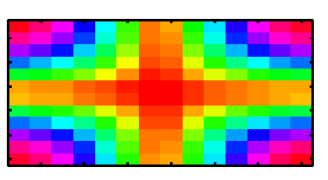

Figure 20: Comparison of some eigenvectors of $\mathrm{T}$ with the corresponding eigenmodes of a room $12 \mathrm{~m}$ wide for $15 \mathrm{~m}$ long. 


\section{References}

[1] A Le Bot. Foundation of statistical energy analysis in vibroacoustics. OUP Oxford, 2015.

[2] R.H. Lyon. Statistical Energy Analysis of Dynamical Systems. M.I.T. Press, 1975 .

[3] A. Culla and A. Sestieri. Is it possible to treat confidentially SEA the wolf in sheep's clothing? Mech. Syst. Signal Proc., 20(6):1372-1399, 2006.

[4] E. Reynders and G. De Roeck. Reference-based combined deterministicstochastic subspace identification for experimental and operational modal analysis. Mech. Syst. Signal Proc., 22(3):617-637, 2008.

[5] E. Reynders. System identification methods for (operational) modal analysis: review and comparison. Archives of Computational Methods in Engineering, 19(1):51-124, 2012.

[6] G. De Sitter, Ch. Devriendt, P. Guillaume, and E. Pruyt. Operational transfer path analysis. Mech. Syst. Signal Proc., 24(2):416-431, 2010.

[7] P. Gajdatsy, K. Janssens, W. Desmet, and H. Van Der Auweraer. Application of the transmissibility concept in transfer path analysis. Mech. Syst. Signal Proc., 24(7):1963-1976, 2010.

[8] A. Oktav, Ç. Yılmaz, and G. Anlaş. Transfer path analysis: Current practice, trade-offs and consideration of damping. Mech. Syst. Signal Proc., 85:760-772, 2017.

[9] X. Chen, D. Wang, and Z. Ma. Simulation on a car interior aerodynamic noise control based on statistical energy analysis. Chinese Journal of Mechanical Engineering, 25(5):1016-1021, 2012.

[10] J. Forssén, S. Tober, A.C. Corakci, A. Frid, and W. Kropp. Modelling the interior sound field of a railway vehicle using statistical energy analysis. Appl. Acoust., 73(4):307-311, 2012.

[11] M. Kassem, C. Soize, and L. Gagliardini. Structural partitioning of complex structures in the medium-frequency range. An application to an automotive vehicle. J. Sound Vibr., 330(5):937-946, 2011.

[12] L. Kovalevsky and R.S. Langley. Automatic recognition of the components of a hybrid FE-SEA model. In Proceedings of the Acoustics 2012 Nantes Conference, Nantes, France, 23 - 27 April 2012, 2012.

[13] L. Gagliardini, L. Houillon, G. Borello, and L. Petrinelli. Virtual SEA - FEAbased modeling of mid-frequency structure-borne noise. Sound and Vibration, $39(1): 22-28,2005$. 
[14] N. Totaro and J.L. Guyader. SEA substructuring using cluster analysis: The MIR index. J. Sound Vibr., 290(12):264-289, 2006.

[15] C. Díaz-Cereceda, J. Poblet-Puig, and A. Rodríguez-Ferran. Automatic subsystem identification in statistical energy analysis. Mech. Syst. Signal Proc., 5455(0):182-194, 2015.

[16] B.S. Everitt, S. Landau, M. Leese, and D. Stahl. Hierarchical clustering. Cluster Analysis, 5th Edition, pages 71-110, 2011.

[17] F.X. Magrans, J. Poblet-Puig, and A. Rodríguez-Ferran. Sub-structuring of mechanical systems based on the path concept. In International Congress and Exposition on Noise Control Engineering, pages 2485-2494. Deutsche Gesellschaft fr Akustik e.V. (DEGA), Aug 2016.

[18] F.X. Magrans, J. Poblet-Puig, and A. Rodríguez-Ferran. The solution of linear mechanical systems in terms of path superposition. Mech. Syst. Signal Proc., $85: 111-125,2017$.

[19] F. X. Magrans. Direct transference applied to the study of room acoustics. J. Sound Vibr., 96(1):13-21, 1984.

[20] F. X. Magrans. Definition and calculation of transmission paths within an SEA framework. J. Sound Vibr., 165(2):277-283, 1993.

[21] M. Van Der Seijs, D. de Klerk, and D. J. Rixen. General framework for transfer path analysis: History, theory and classification of techniques. Mech. Syst. Signal Proc., (68-69):217-244, 2015.

[22] E.E. Ungar. Fundamentals of statistical energy analysis of vibrating systems. Technical report, DTIC Document, 1966.

[23] F. Fahy and P. Gardonio. 7 - acoustic coupling between structures and enclosed volumes of fluid. In P. Gardonioand F. Fahy, editor, Sound and Structural Vibration (Second Edition), pages 403-448. Academic Press, Oxford, second edition edition, 2007.

[24] L. Gagliardini and J.L. Guyader. The coupling eigenvalues method: a new basis for the study of vibro-acoustics coupled systems. In NOVEM, Lyon, France, 2000.

[25] G Maidanik. Response of coupled dynamic systems. J. Sound Vibr., 46(4):561$583,1976$. 